\title{
A MULTI-CHANNEL CHANNEL-OPTIMIZED SCHEME FOR EZW USING RATE-DISTORTION FUNCTIONS
}

\author{
Jen-Chang Liu ${ }^{\ddagger \dagger}$, Wen-Liang Hwang ${ }^{\dagger}$,Wen-Jyi Hwang*, and Ming-Syan Chen ${ }^{\ddagger}$ \\ Department of Electrical Engineering, National Taiwan University ${ }^{\ddagger}$ \\ Institute of Information Science, Academia Sinica, Nankang, Taipei, Taiwan, R.O.C. ${ }^{\dagger}$ \\ Department of Electrical Engineering, Chung Yuan Christian University* \\ ${ }^{\dagger}$ Email : whwang@iis.sinica.edu.tw ${ }^{\dagger}$ Fax: 886-2-27824814
}

\begin{abstract}
We develop a multi-channel channel-optimized scheme for EZW image compression in a noisy transmission environment. A block-based modification for EZW is applied to improve the robustness of EZW, and to produce several coded bitstreams for transmission over multiple channels with different noise conditions. Then the respective channel noise is considered in the ratedistortion analysis, and the resultant rate-distortion functions are used for optimal bit allocation among the coded bitstreams. The case of burst noise is analyzed as an example.
\end{abstract}

\section{INTRODUCTION}

The modern communication system mainly consists of the source coding and the channel coding. These two parts are usually designed separately because of Shannon's separation principle [1]. The separated design of source coding from channel coding can be potentially inefficient in practice. For example, the Embedded $\mathrm{Ze}$ rotree Wavelet (EZW) coder [2] is known to be the state-of-art image compression algorithm, but even a single bit error occurring in the transmitted bitstream will drastically affect the whole decoded image quality. In this case, the channel coding has to be designed to guarantee an almost error-free performance, which will cost lots of redundant bits. Therefore, the joint consideration of source and channel coding may be necessary and useful [3]. One class of joint source-channel coding is the channel-optimized source coding, which considers the channel properties in the source coding design. According to the idea of channel-optimized source coding, we propose a multi-channel channel-optimized source coding adaptation for EZW using the rate-distortion functions.

Because EZW lacks the ability of error-resilience, we first adopt the block-based modification for EZW
[4], which will improve the robustness of the original EZW. The block-based EZW coding will produce several bitstreams according to the block partitions, and these bitstreams can be assigned to different channels with respective noise conditions and cost function(for example, the high performance channel will cost more). Then we can compute the rate-distortion function of each block partition using the statistics of the channel properties. Hence, we can optimally allocate the bit budget to each block partition using the calculated rate-distortion functions.

\section{THE MULTI-CHANNEL CHANNEL-OPTIMIZED SOURCE CODING FRAMEWORK}

As we stated earlier, we have adopted the block-based modification for EZW [4] to improve the robustness. An image can be divided into small square blocks, and we can arrange the blocks into separated partitions, in which the blocks are then encoded using the original EZW algorithm to produce independent bitstreams. The choice of partitions is another issue; for example, we can arrange the blocks containing edges in one partition and send it through a reliable channel. In each partition, the variable-length bitstreams of the blocks are arranged using the EREC (Error-Resilient Entropy Code) [5] scheme to improve the ability of synchronization, and are interleaved to form a single bitstream. Then, the coded bistreams of all partitions are sent through their assigned channels. Therefore, we have a multi-channel framework as shown in Fig. 1.

In the above multi-channel framework, we want to optimally allocate the bit budget to each block in the sense of less overall distortion and cost. For each block in our block-based EZW scheme, we can measure its rate-distortion function. By assigning $n$ bits for block $i$, then we can calculate the MSE (mean square error) between the original and decoded wavelet coefficients 
as its distortion $D_{i}(n)$. The bit allocation problem of assigning $b_{i}$ bits to block $i, i=1 \ldots K$, with total bit budget $R$, and the cost function $C_{i}(n)$ of the channel responsible for block $i$, can be formulated as follows.

$$
\begin{array}{r}
\min \sum_{i=1}^{K} D_{i}\left(b_{i}\right)+C_{i}\left(b_{i}\right) \\
\text { subject to } \quad \sum_{i=1}^{K} b_{i} \leq R
\end{array}
$$

Conventional dynamic programming skill can be used to solve the above problem.

An desired property of the above bit allocation procedure is that, with the increasing of total bit budget $R$, the allocated bits to each block are also monotonically increasing. To prove this fact, we refer to two results from [7]. For simplicity, the cost term is removed in the following derivation because it can be included in the distortion term. The first theorem is a result from Lagrange multiplier method.

Theorem 1: For any $\lambda \geq 0$, the solution $b_{i}^{*}(\lambda), i=$ $1 . . K$, to the unconstrained problem

$$
\min _{\left\{b_{i}, i=1 \ldots K\right\}} \sum_{i=1}^{K} D_{i}\left(b_{i}\right)+\lambda \sum_{i=1}^{K} b_{i}
$$

is also the solution to the constrained problem (1), with the constraint $R=\sum_{i=1}^{K} b_{i}^{*}(\lambda)$.

For a given $\lambda$; solution to (2) can be obtained by minimizing each term of the sum in (2) separately. We need one lemma from [7] for our proof.

Lemma 1: Let $D(b)$ be a real-valued function over some bounded and closed domain $Z$ in the real line. Let $b_{1}$ be a solution to

$$
\min _{b \in Z}\left\{D(b)+\lambda_{1} b\right\}
$$

and let $b_{2}$ be a solution to

$$
\min _{b \in Z}\left\{D(b)+\lambda_{2} b\right\}
$$

then,

$$
\left(\lambda_{1}-\lambda_{2}\right)\left(b_{1}-b_{2}\right) \leq 0
$$

for any function $D(b)$.

We prove our theorem as follows.

Theorem 2: For bit budgets $R_{1} \geq 0$ and $R_{2} \geq$ 0 , let their corresponding optimal solutions to (1) be $\left\{b_{11}, b_{12}, \ldots, b_{1 K}\right\}$ and $\left\{b_{21}, b_{22}, \ldots, b_{2 K}\right\}$. If $R_{1}<R_{2}$, then $b_{1 i} \leq b_{2 i}, i=1$...K.

Proof: Let the solution $\lambda$ corresponding to $R_{1}$ and $R_{2}$ be $\lambda_{1}$ and $\lambda_{2}$, respectively. Since $R_{1}<R_{2}$, there exits some block $m$, such that $b_{1 m} \leq b_{2 m}$. From Lemma 1,

$$
\left(\lambda_{1}-\lambda_{2}\right)\left(b_{1 m}-b_{2 m}\right) \leq 0
$$

we could derive that $\lambda_{1} \geq \lambda_{2}$. Apply Lemma 1 and $\lambda_{1} \geq \lambda_{2}$ to other blocks, we could derive that

$$
b_{1 i} \leq b_{2 i}, i=1 \ldots K . \quad \triangle
$$

The above theorem provides the progressive property to each block.

\section{RATE-DISTORTION FUNCTIONS CONSIDERING BURST NOISE}

In this section, we analyze the rate-distortion functions by considering transmission over channels with burst noise. The Gilbert-Elliot(G-E) model [6] is used for burst noise simulation. The G-E model is shown as Fig. 2, in which there are two states: $G$ (Good) state is almost error free, and has probability of error $(1-k)$; $B$ (Bad or Burst) state represents the burst errors, and has probability of error $(1-h)$. Note that $(1-k) \ll(1-$ $h)$. The transition probabilities $P(B \mid G)$ and $P(B \mid G)$ are $p$ and $q$, respectively.

Let $D(n)$ be the distortion of encoding and decoding $n$ bits for an image block without error in transmitting these $n$ bits. We can formulate the expected rate-distortion function adapted to any channel noise condition as follows,

$$
D^{*}(n)=\sum_{j=1}^{n} P(j) D(j-1)+P^{0}(n) D(n),
$$

where $P(j)$ is the probability that the first error occurred at the $j$-th bit, and $P^{0}(n)$ is the probability that all encoded $n$ bits are error free. $P(j)$ and $P^{0}(n)$ are dependent on the channel noise model. Since $\sum_{j=1}^{n} P(j)+$ $P^{0}(n)=1$, we call $D^{*}(n)$ the expected distortion while transmitting the encoded $n$ bits in a noisy channel with probability distribution $P(j), j=1 \ldots n$ and $P^{0}(n)$.

The calculation of $P(j)$ and $P^{0}(n)$ for the G-E model is presented in [4].

We can replace the $D_{i}\left(b_{i}\right)$ term in the constrained bit allocation problem (1) by the $D_{i}^{*}\left(b_{i}\right)$ term computed for each block $i$ using the above calculation process. Applying any optimal bit allocation strategy will produce the ideal bit allocation result over the noisy channels.

\section{EXPERIMENT RESULTS}

In this section, we apply our method to the burst noise channel as an experiment. We demonstrate the simpliest case of one channel. The G-E model is used to simulate the burst noise as in Fig. 2, where there are totally 4 parameters. It is difficult and less meaningful to control these 4 parameters and describe the results 
using these parameters. We adopted the simplification of the G-E model in [9].

The simplified G-E model replaced the original 4 parameters with 3 more meaningful parameters. The first parameter is $\bar{\varepsilon}$, the average $B E R$ of the channel. Note that

$$
\bar{\varepsilon}=\frac{q(1-k)+p(1-h)}{p+q} .
$$

The second parameter is $\vec{b}$, the average burst length, i.e., the average number of times stayed in the $\mathrm{B}(\mathrm{Bad})$ state. Note that

$$
\bar{b}=\frac{1}{q}
$$

The third parameter is $p_{1}$, the duty cycle, or the steady-state probability of being in the B state. Note that

$$
p_{1}=\frac{p}{p+q}
$$

These three parameters $\bar{\varepsilon}, \bar{b}$, and $p_{1}$ have more direct meanings to characterize the burst noise of the channel, than the original four parameters. Since we have one less degree of freedom than the original G-E model, we have to introduce the following relation,

$$
1-k=\bar{\varepsilon} p_{1} \text {. }
$$

This added relation ensures that the simplified G-E model is able to describe the dense(low duty cycle, and high intensity, i.e., high $\frac{1-h}{1-k}$ ), and diffuse(large duty cycle, and low intensity) conditions [9].

In Fig. 3, we compared the performance our methods with the equal bit allocation method [8], and the original EZW over burst noise channels with fixed $p_{1}$ and $\bar{b}$. Note that the conditions $p_{1}=0.5$ and $\bar{b}=2$ represent a case of the fast fading burst noise. It is obvious that our method have better performance than the other two methods. The performance is insignificant at $\mathrm{BER}=10^{-2}$ because of the high bit error rates.

In Fig. 4, we showed the performance results with fixed $p_{1}=0.5$ and $\bar{b}=12.5$, which represent the slow fading burst noise. Our method still has better performance than the others.

As an example, we simulate a channel with the burst noise model with parameters $p=0.0001, q=$ $0.07, k=1, h=0.3$, and Lena images coded with the original EZW and our proposed method respectively at $1 b p p$ rate, are sent through the noisy channel. The decoded results are shown in Fig. 5(a) and Fig. 5(b). It is clear that our method is more robust than the original EZW.

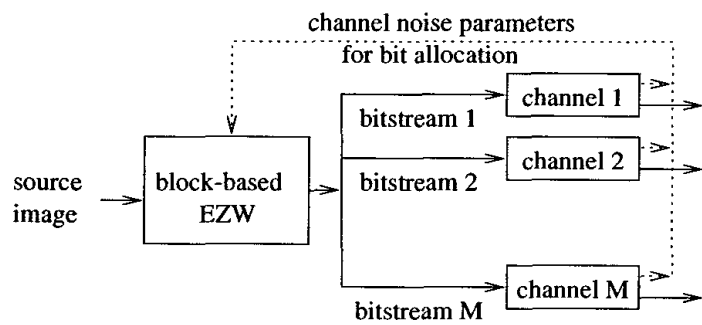

Figure 1: The multi-channel channel-optimized source coding framework.

\section{REFERENCES}

[1] C.E. Shannon, "Coding Theorems for a Discrete Source with a Fidelity Criterion", IRE National Convention Record, Part 4, pp. 142-163, 1959.

[2] Jerome M. Shapiro, "Embedded Image Coding Using Zerotrees of Wavelet Coefficients", IEEE Trans. Signal Processing, vol. 41, no. 12, pp. 3445-3462, Dec. 1993.

[3] Igor Kozintsev and Kannan Ramchandran, "Robust Image Transmission Over Energy-Constrained Time-Varying Channels Using Multiresolution Joint Source-Channel Coding", IEEE Trans. Signal Processing, Vol. 46, no. 4, pp. 10121026, Apr. 1998.

[4] J.C. Liu, W.L. Hwang, W.J. Hwang, and M.S. Chen, "Robust Block-Based EZW Image Compression with Channel Noise Optimized Rate-Distortion Functions", Proc. of ICIP'g9, 1999.

[5] D. W. Redmill and N. G. Kingsbury, "The EREC: An ErrorResilient Technique for Coding Variable-Length Blocks of Data", IEEE Trans. Image Processing, vol. 5, no. 4, pp. 565-574, Apr. 1996.

[6] E. N. Gilbert, "Capacity of a burst-noise channel", Bell Sys. Tech. J. 39, pp. 1253-1266, 1960.

[7] Y. Shoham, and A. Gersho, "Efficient Bit Allocation for an Arbitrary Set of Quantizers", IEEE Trans. Acoustic, Speech, and Signal Processing, vol. 36, no. 9, pp. 1445-1453, Sep. 1988.

[8] Charles. D. Creusere, "A New Method of Robust Image Compression Based on the Embedded Zerotree Wavelet Algorithm", IEEE Trans. Image Processing, vol. 6, no. 10, pp. 1436-1442, Oct. 1997.

[9] Robert H. Deng, "Hybrid ARQ Schemes for Point-toMultipoint Communication Over Nonstationary Broadcast Channels", IEEE Trans. Communication, vol. 41, no. 9, pp. 1379-1387, Sep. 1993. 


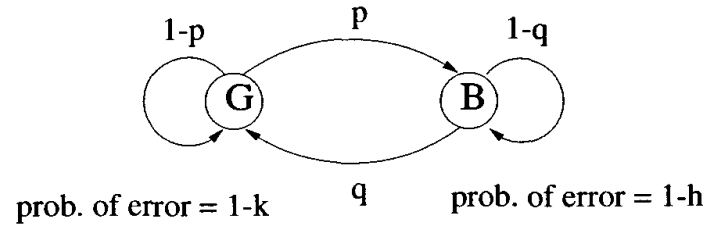

Figure 2: The Gilber-Elliot burst noise model.

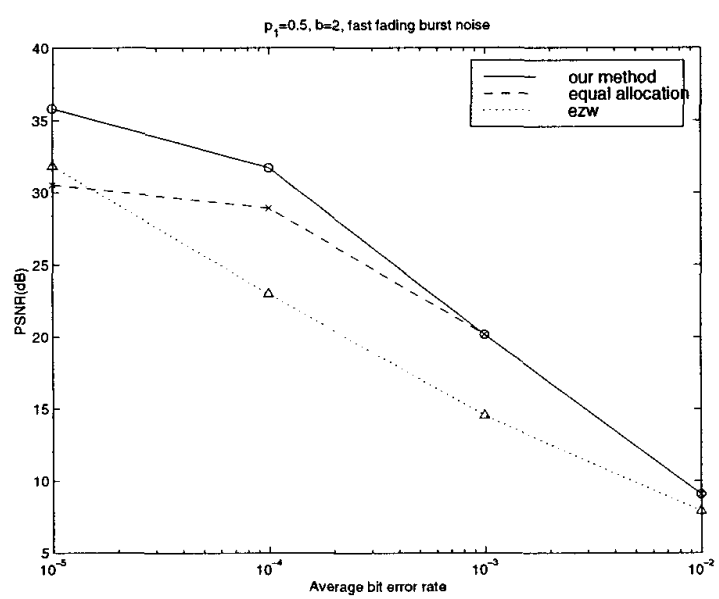

Figure 3: The PSNR performance of various compression schemes in fast fading burst noise channel.

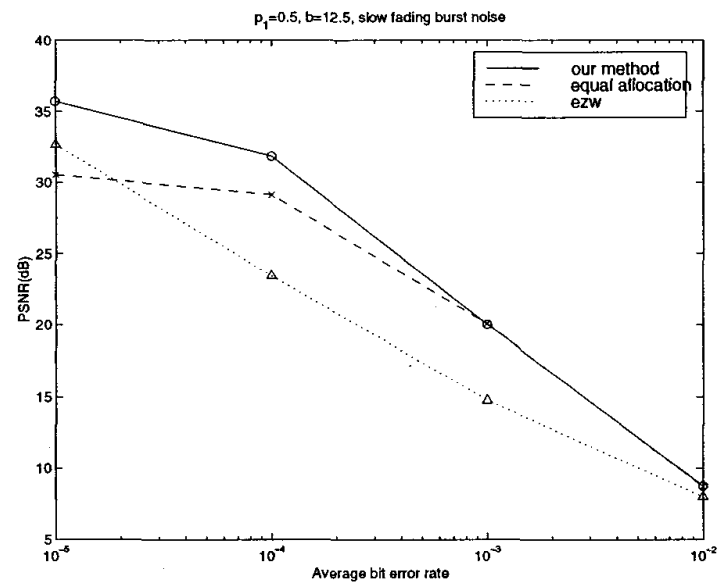

Figure 4: The PSNR performance of various compression schemes in slow fading burst noise channel.

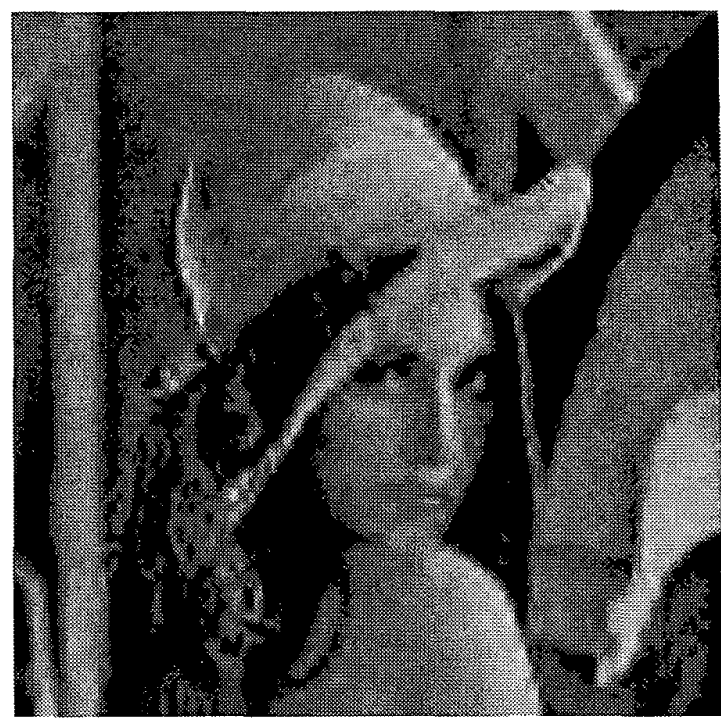

(a)

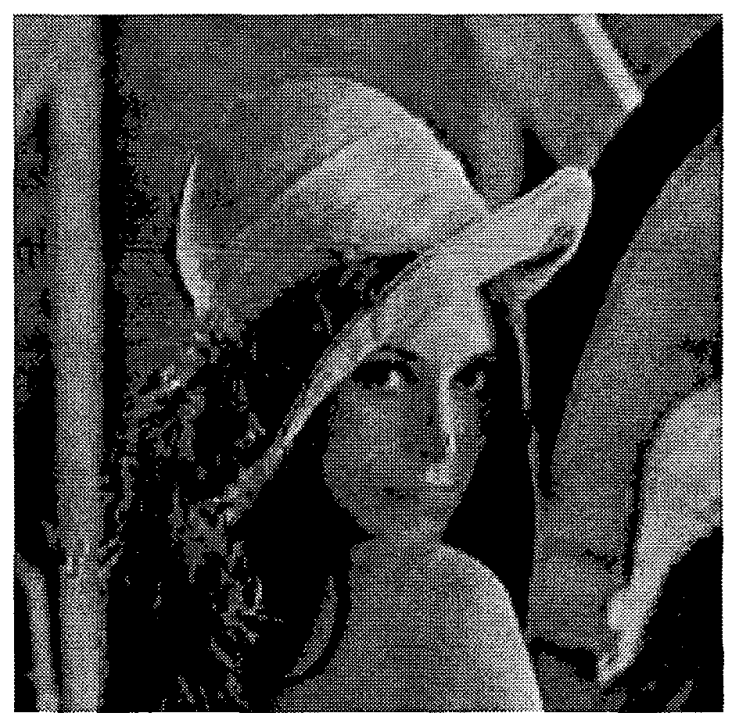

(b)

Figure 5: Decoded result of Lena image through a burst noise channel. (a) The original EZW. (b) The proposed method. 